TRANSACTIONS OF THE

AMERICAN MATHEMATICAL SOCIETY

Volume 358, Number 5, Pages 2161-2181

S 0002-9947(05)03746-3

Article electronically published on May 26, 2005

\title{
DUAL DECOMPOSITIONS OF 4-MANIFOLDS II: LINEAR INVARIANTS
}

\author{
FRANK QUINN
}

\begin{abstract}
This paper continues the study of decompositions of a smooth 4 -manifold into two handlebodies with handles of index $\leq 2$. Part I (Trans. Amer. Math. Soc. 354 (2002), 1373-1392) gave existence results in terms of spines and chain complexes over the fundamental group of the ambient manifold. Here we assume that one side of a decomposition has larger fundamental group, and use this to define algebraic-topological invariants. These reveal a basic asymmetry in these decompositions: subtle changes on one side can force algebraic-topologically detectable changes on the other. A solvable iteration of the basic invariant gives an "obstruction theory" using lower commutator quotients. By thinking of a 2-handlebody as essentially determined by the links used as attaching maps for its 2-handles, this theory can be thought of as giving "ambient" link invariants. The moves used are related to the grope cobordism of links developed by Conant-Teichner, and the CochranOrr-Teichner filtration of the link concordance groups. The invariants give algebraically sophisticated "finite type" invariants in the sense of Vassilaev.
\end{abstract}

\section{INTRODUCTION}

A dual decomposition of a 4-manifold is a description as a union of two handlebodies, each with handles of index $\leq 2$. In Part I Q we gave existence results for these in terms of spines and chain complexes over the fundamental group of the ambient manifold; here we incorporate refinements of the fundamental group. Suppose $\Gamma \rightarrow \pi_{1} N$ is a homomorphism. Then a " $\Gamma$ decomposition of $N$ " is a dual decomposition $N=M \cup W$ together with a factorization $\pi_{1} W \rightarrow \Gamma \rightarrow \pi_{1} N$. We study these using linear invariants, particularly the cellular chains of the $\Gamma$ cover of $W$. We will not consider bilinear invariants, such as signatures, that incorporate intersection information.

There is a basic asymmetry in the behavior of dual decompositions: subtle changes of one side can force algebraic-topologically detectable changes on the other. We use this to probe the subtle changes. If we think of $M$ as being primarily determined by the link giving the attaching map of the 2-handles, then we see invariants of $W$ as giving information about this link. Or if we think of $M$ as a surgery presentation of the 3-manifold $\partial M$, then invariants of $W$ give information about this 3-manifold. This information is closely related to classical link and 3-manifold invariants, and to some "quantum" invariants. In terms of links these invariants are local and relative, measuring perturbations of a given link, inside a fixed ambient

Received by the editors December 10, 2001 and, in revised form, May 11, 2004.

2000 Mathematics Subject Classification. Primary 57R65, 57M25.

This work was partially supported by the National Science Foundation.

(C)2005 American Mathematical Society Reverts to public domain 28 years from publication 
4-manifold. However they are better defined and behaved than most global link invariants. They also have direct connections to a more mainstream topic than classical link theory, namely handlebody theory of smooth 4-manifolds.

This paper develops the conceptual and formal structure of linear invariants of $\Gamma$ decompositions. The main geometric results, for instance, show that when properly defined the invariants are all realized. The theory is somewhat elaborate, so in this paper we are content with a careful development that should clarify the possibilities and limitations. Work on computation, explicit examples, or exact relations with other invariants should perhaps wait for a clearer idea of a job to be done.

We describe the invariants in more detail. If a decomposition $N=M \cup W$ is deformed in a way that does not change the $Z\left[\pi_{1} N\right]$ chains of $M$ (up to chain homotopy), then duality shows that the $Z\left[\pi_{1} N\right]$ chains of $W$ do not change either. On this level there are no invariants. However if there is a factorization $\pi_{1} W \rightarrow$ $\Gamma \rightarrow \pi_{1} N$, then the $Z[\Gamma]$ chains may vary within the constraint that the $Z\left[\pi_{1} N\right]$ reduction is fixed. The general setting is a homomorphism of groups $\Lambda \rightarrow \Gamma$, and free based chain complexes over $Z[\Lambda]$ with a fixed $Z[\Gamma]$ reduction. If $M \cup W$ is a decomposition with $\pi_{1} W \rightarrow \Lambda \rightarrow \Gamma \rightarrow \pi_{1} N$ given, then the $\Lambda \rightarrow \Gamma$ "chain invariant" is defined simply as an appropriate equivalence class of such chain data. By design it is unchanged by $\Lambda$ deformations.

We explain how chain invariants can be seen as obstructions. Suppose $M_{0} \cup$ $W_{0}$ is a decomposition with a factorization $\pi_{1} W_{0} \rightarrow \Lambda \rightarrow \Gamma \rightarrow \pi_{1} N$. The $\Gamma$ deformation move described in Section 3 gives a new $\Gamma$-decomposition $M_{1} \cup W_{1}$, together with a canonical simple chain equivalence of the chains of $W_{0}$ and $W_{1}$, with $Z[\Gamma]$ coefficients. Suppose it should happen that the canonical factorization $\pi_{1} W_{1} \rightarrow \Gamma \rightarrow \pi_{1} N$ lifts to $\Lambda$. Then the $\Lambda$ chains of $W_{i}$ together with the canonical equivalence over $\Gamma$ provide the data needed to define the $\Lambda \rightarrow \Gamma$ chain invariant. Note that if the deformation were actually a $\Lambda$ deformation, then the complexes would be equivalent over $Z[\Lambda]$ and the invariant of $W_{1}$ would be the same as that of $W_{0}$. Therefore the invariant gives an obstruction to $M_{1}$ together with this particular lift $\pi_{1} W_{1} \rightarrow \Lambda$ to come from a $\Lambda$ deformation.

The factorization $\pi_{1} W \rightarrow \Lambda$ in the previous paragraph presents a problem. The chain invariant is not defined unless a factorization exists, and then may depend on which one is used. However we observe that given $\pi_{1} W \rightarrow \Gamma$ there is a particular $\Lambda$ for which canonical factorizations exist. This is $\Gamma_{1}(W)=\pi_{1} W / \operatorname{ker}\left(\pi_{1} N \rightarrow \Gamma\right)^{(1)}$, where the superscript "(1)" in the denominator denotes the commutator subgroup. $\Gamma_{1}(W)$ is an abelian extension of $\Gamma$, and the chain invariant in this case is an analog of the classical abelian link invariants.

The abelian extension construction can be iterated to get an "obstruction theory" for invariants using solvable extensions of $\Gamma$. Specifically we define $\Gamma_{n}(W)$ to be $\pi_{1} W$ divided by the $n$-fold iterated commutator of the kernel of the homomorphism to $\Gamma$. Suppose two decompositions have the same $n$-fold solvable extension $\Gamma_{n}\left(W_{0}\right) \simeq \Gamma_{n}\left(W_{1}\right)$, and the same chains over this extension. Then there is a canonical isomorphism $\Gamma_{n+1}\left(W_{0}\right) \simeq \Gamma_{n+1}\left(W_{1}\right)$, and we can compare the $\left(\Gamma_{n+1}, \Gamma_{n}\right)$ chain invariants. If these agree, then the $\Gamma_{n+1}$ chains are the same, and we have the data needed to continue to the next stage. It is these invariants that are related to the Cochran-Orr-Teichner filtration of link concordance [COT].

The paper is organized as follows: Section 2 gives $\Gamma$ versions of the chain and spine realization theorems of Part I. Section 3 describes in detail the move used 
in deformations. This is a refinement of the move used in Part I. There is also a generalization using capped gropes which is the geometric move appropriate for solvable extensions. Section 4 defines $(\Lambda, \Gamma)$ chain invariants and suggests a possible reformulation using algebraic $K$-theory of noncommutative localizations. In Section 5 we state relative versions of the realization theorems, and give proofs. In Section 6 the general theory is specialized to the case of abelian extensions, then iterated to give the obstruction theory for solvable extensions.

\section{ReAlization}

In this section $N$ is a closed smooth 4-manifold. Versions in which $N$ may have boundary are given in Section 4. Fix a homomorphism $\Gamma \rightarrow \pi_{1} N$. A $\Gamma$ decomposition of $N$ is a dual decomposition $N=M \cup W$ together with a factorization $\pi_{1} W \rightarrow \Gamma \rightarrow \pi_{1} N$, so that $\pi_{1} W \rightarrow \Gamma$ is onto. Note that for a $\Gamma$ decomposition to exist $\Gamma$ must be finitely generated and $\Gamma \rightarrow \pi_{1} N$ must be onto, so we assume these conditions hold as well.

2.1 Theorem ( $\Gamma$-Chain Realization). Suppose $D_{*}$ is a finitely generated based free chain complex over $Z[\Gamma]$ with a chain map $f: D_{*} \otimes Z\left[\pi_{1} N\right] \rightarrow C_{*}\left(N ; Z\left[\pi_{1} N\right]\right)$. Then there is a $\Gamma$ decomposition $\left(N=M \cup W, \pi_{1} W \rightarrow \Gamma\right)$ realizing $(D, f)$ if and only if $D$ is homologically 2-dimensional and $H_{0}(D ; Z[\Gamma]) \rightarrow H_{0}\left(N ; Z\left[\pi_{1} N\right]\right)(=Z)$ is an isomorphism.

"Realizing $(D, f)$ " means there is a simple equivalence $g: D_{*} \rightarrow C_{*}(W ; Z[\Gamma])$ so that the diagram

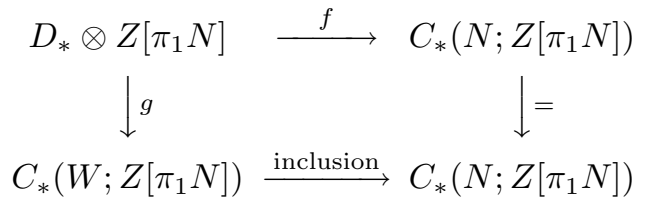

commutes up to chain homotopy. Other terms, e.g. "homologically 2-dimensional" are defined in Part I.

2.2 $\Gamma$-spine realization. Theorem 2.1 describes the chain complexes that can be realized. In the next statement the chains are held fixed and the spine is varied using $\Gamma$ deformations. Deformations are described in detail in Section 3; for the next statement we need the following:

(1) $\Gamma$ deformations of $M$ are defined for codimension-0 submanifolds $M \subset N$ with a factorization $\pi_{1}(N-M) \rightarrow \Gamma \rightarrow \pi_{1} N$. In particular if $N=M \cup W$, $\pi_{1} W \rightarrow \Gamma \rightarrow \pi_{1} N$ are a $\Gamma$ decomposition in the sense of the introduction, then $M$ can be $\Gamma$ deformed.

(2) The output is another $\Gamma$ decomposition $M^{\prime} \cup W^{\prime}, \pi_{1} W^{\prime} \rightarrow \Gamma \rightarrow \pi_{1} N$.

(3) There is a canonical simple chain equivalence $C_{*}(W ; Z[\Gamma]) \stackrel{\sim}{\longrightarrow} C_{*}\left(W^{\prime} ; Z[\Gamma]\right)$, whose $Z\left[\pi_{1} N\right]$ reduction chain homotopy commutes with the inclusions to $C_{*}\left(N ; Z\left[\pi_{1} N\right]\right)$.

We show that the $W$ part of the decomposition can change fairly arbitrarily within these constraints. What makes this interesting is that the changes in $M$ are more subtle: the spine is unchanged up to homotopy 2-deformation, for instance. 
$\Gamma$-Spine Realization Theorem. Suppose $N=M \cup W$ is a dual decomposition, $K \rightarrow N$ is a 2-complex, and factorizations $\pi_{1} W \rightarrow \Gamma \rightarrow \pi_{1} N$ and $\pi_{1} K \rightarrow \Gamma \rightarrow \pi_{1} N$ are given. Then there is a $\Gamma$ deformation of $M$ to a decomposition $M^{\prime} \cup W^{\prime}$ so that the spine of $W^{\prime}$ realizes $K$ up to homotopy deformation, if and only if there is a simple chain equivalence $C_{*}(K ; Z[\Gamma]) \rightarrow C_{*}(W ; Z[\Gamma])$ whose $Z\left[\pi_{1} N\right]$ reduction chain-homotopy commutes with the inclusions.

In fact the simple chain equivalence is realized in the sense that the diagram

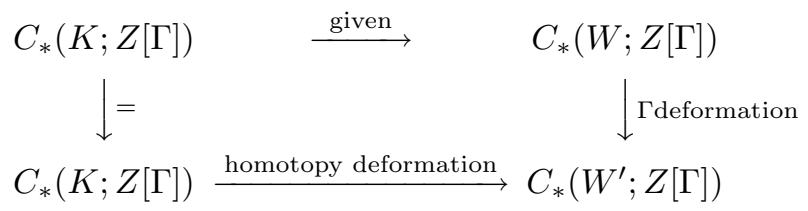

chain homotopy commutes.

Recall from Part I that a homotopy deformation of 2-complexes is a sequence of moves, either expansions or collapses of 1- or 2-cells, or changes of attaching map of 2-cells by homotopy. A homotopy deformation determines a simple homotopy equivalence, which in turn induces a simple chain equivalence (with any coefficients). The converses are not true: a simple chain equivalence between cellular chains of spaces is rarely induced by a map, and we do not expect simple homotopy equivalences of 2-complexes to always come from homotopy 2-deformations. This last statement is the "Andrews-Curtis conjecture", and though expected to be false, it is still open.

2.3 $\Gamma_{n}$-spine realization. Grope deformations are refinements that geometrically encode some fundamental group information. They therefore apply only to certain factorings of $\pi_{1} W$. Suppose $\pi_{1} W \rightarrow \Gamma$ as usual, and as in the Introduction we define

$$
\Gamma_{n}(W)=\left(\pi_{1} W\right) / \operatorname{ker}\left(\pi_{1} W \rightarrow \Gamma\right)^{(n)} .
$$

Here $K^{(n)}$ is the $n$-fold iterated commutator subgroup defined by $K^{(0)}=K$ and $K^{(n)}=\left[K^{(n-1)}, K^{(n-1)}\right]$. Grope deformations are defined in 3.5, and in 3.6 we show that a $\Gamma$ grope deformation of height $n$ is a particular case of standard $\Gamma_{n-1}(W)$ deformation. The converse is not true, but there are enough of them to change skeleta in the same way.

Theorem. Suppose $N=M \cup W$ is a dual decomposition with factorization $\pi_{1} W \rightarrow$ $\Gamma \rightarrow \pi_{1} N$. Then the conclusions of the Theorem in 2.2 for the factorization $\pi_{1} W \rightarrow$ $\Gamma_{n} W \rightarrow \pi_{1} N$ hold with " $\Gamma$ grope deformation of height $n+1$ " replacing " $\Gamma_{n} W-$ deformation".

The standard deformations are grope deformations of height 1 , so this statement includes 2.2 as the special case $n=0$. As in 2.2 the deformations realize the chain equivalence.

\section{T-DEFORMATIONS}

In this section we suppose $N$ is a compact smooth 4-manifold with boundary divided into pieces $\partial N=\partial_{0} M \cup \partial_{0} W$. At this point $M$ and $W$ are not defined; the notations for the pieces of $\partial N$ are for later convenience. Fix a homomorphism $\Gamma \rightarrow \pi_{1} N$, and sometimes a further homomorphism $\Lambda \rightarrow \Gamma$. We consider extensions of the boundary decomposition to dual decompositions $N=M \cup W$, together 


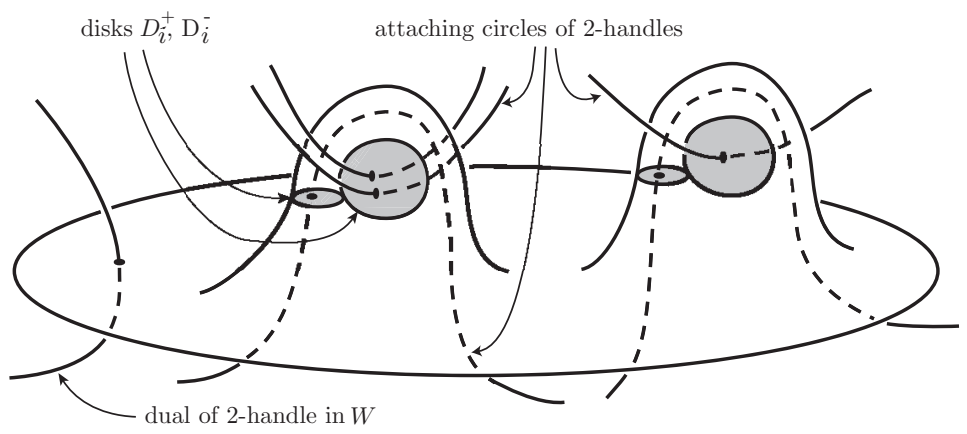

FiguRE 1.

with a factorization $\pi_{1} W \rightarrow \Gamma \rightarrow \pi_{1} N$. Explicitly, "extension" of the boundary decomposition means $M \cap \partial N=\partial_{0} M, W \cap \partial N=\partial_{0} W$, and the significance of "dual decomposition" is that we are given handlebody structures with handles of index $\leq 2$ on the pairs $\left(M, \partial_{0} M\right)$ and $\left(W, \partial_{0} W\right)$. The basic $\Gamma$ and $(\Lambda, \Gamma)$ deformation moves are described from several viewpoints in 3.1-3.3. Standard structure (chain maps, etc.) is derived in 3.4, and the grope version is described in 3.5-3.7.

A $\Gamma$ deformation is a sequence of $\Gamma$ decompositions of $N$, each obtained from the previous one either by ordinary handle moves in $M$ and $W$, or by the move described next:

3.1 The data. As above we have $N=M \cup W$ and a factorization $\pi_{1} W \rightarrow \Gamma \rightarrow$ $\pi_{1} N$. The data for a $\Gamma$-deformation of $M$ is:

(1) a compact orientable surface $\Sigma$ with boundary $S^{1}$ and a standard hyperbolic basis of embedded curves;

(2) an embedding $\Sigma \cup D_{*}^{ \pm} \rightarrow \partial_{1} M^{(1)}$, where $D_{*}^{ \pm}$are 2-disks attached on the basis curves and $\partial_{1} M^{(1)}$ is the level in the handlebody structure on $M$ between the 1-handles and the 2-handles;

(3) $\Sigma$ is disjoint from the 2-handles of $M$, so lies in $\partial_{1} W$. This map $\Sigma \rightarrow W$ lifts to the $\Gamma$ cover of $W$;

(4) the disks are disjoint from the 2-handles of $W$; and

(5) $\Sigma$ intersects a single 2-handle of $W$, and intersects the dual core of that handle in a single point.

If further a pair of groups $\Lambda \rightarrow \Gamma \rightarrow \pi_{1} N$ is given and $\pi_{1} W$ lifts to $\Lambda$, then the data for a $(\Lambda, \Gamma)$-deformation is the above and

(6) the boundary curve of $\Sigma$ lifts to the $\Lambda$ cover of $W$.

This data is illustrated in Figure 1. In standard $4 \mathrm{~d}$ terminology (cf. [FQ]) $\Sigma \cup D_{*}^{ \pm}$is a disk-like capped surface. The caps intersect 2-handles of $M$, while the body has a single point of intersection with (cocores of) handles of $W$. Note that "2-handles of $W$ " are by definition attached to lower handles of $W$. The duals of these are handles attached to the complement (more specifically to $\partial_{1} M^{(1)}$ ). The intersection with $\Sigma$ is with the attaching circle of one of these dual handles.

3.2 The move as seen from $M$. Suppose we have the data specified above, and denote by $A$ the handle of $W$ intersecting $\Sigma$. We denote the dual handle by $A^{*}$, so it is the attaching circle of $A^{*}$ that intersects $\Sigma$ in a point. The move changes 

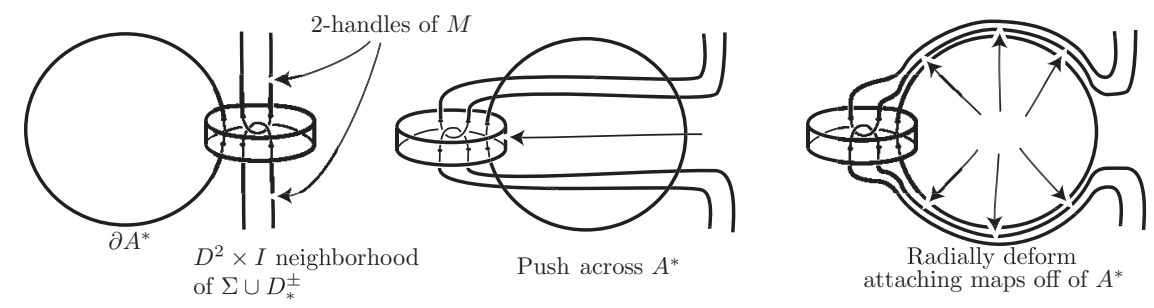

FIGURE 2.
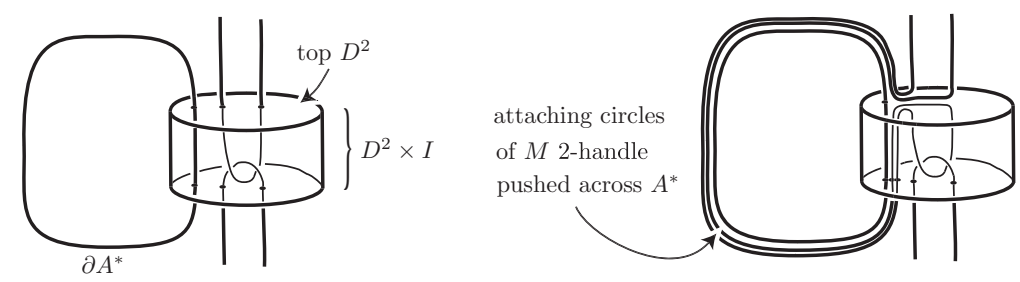

FIGURE 3.

$M \cup A^{*}$ by handle moves and the result of the move $\left(M^{\prime}\right)$ is the complement of $A^{*}$ in the resulting handlebody structure. The handle moves are obtained by pushing a neighborhood of $\Sigma \cup D_{*}^{ \pm}$over $A^{*}$. In 3.2.1 we describe this in a symmetric form, then in 3.2 .2 a less symmetric form that may be easier to see.

3.2.1 The symmetric view. A neighborhood of $\left(\Sigma \cup D_{*}^{ \pm}, \partial \Sigma\right)$ in $\partial_{1} M^{(1)}$ is isomorphic to a ball $\left(D^{2} \times I, S^{1} \times I\right)$. The attaching circle of $A^{*}$ intersects this in an arc $\{p\} \times I$. Push this ball across $A^{*}$ through the center to the other side, dragging the attaching maps of $M$ 2-handles along. After this the attaching maps miss the center of the core of $A^{*}$, so we can deform them radially off $A^{*}$. This sequence of moves is shown in Figure 2.

This gives an isotopy of attaching maps of 2-handles of $M$, in the boundary of $M^{(1)} \cup A^{*}$. Use this to do handle moves in the handlebody $M \cup A^{*}$. This changes the handlebody structure but does not change the manifold. In particular it is still embedded in $N$ with complement $W-A$. Define $M^{\prime}$ to be the complement of $A^{*}$ in this new handlebody, and define $W^{\prime}$ to be (the closure of) the complement of $M^{\prime}$ in $N$. Note that $W^{\prime}$ is given as $W-A$ union with the repositioned $A$.

3.2.2 The asymmetric view. We now give a less symmetric but more elementary description of the deformation. Consider the top $D^{2}$ of the $D^{2} \times I$ neighborhood of the capped surface. This disk intersects the attaching map of $A^{*}$ in a single point, and many attaching maps of $M$ 2-handles. Do handle moves of the $M$ 2-handles across $A^{*}$ to make them disjoint from the disk. This involves pushing along arcs in the disk, then over $A^{*}$. This operation is shown in Figure 3.

Again we define $M^{\prime}$ to be the resulting handlebody, with $A^{*}$ omitted. Comparing Figures 2 and 3 shows the two operations are isotopic, so this gives the same result as before. Also we could do the same thing with the lower $D^{2}$ in the ball neighborhood. This is also isotopic to the symmetric version, so we additionally see that using the upper or lower disks yield isotopic results. 
3.3 The move as seen from $W$. The surface $\Sigma$ intersects the core of the handle $A^{*}$ in a point, so intersects $A^{*}$ in a disk. Deleting the interior of this disk leaves a surface $\Sigma_{0}$ with two boundary circles. $\Sigma_{0}$ is disjoint from the interiors of all 2-handles so it also lies in $\partial_{1} W^{(1)}$, the level between the 1- and 2-handles of $W$. In this level the new boundary circle in $\Sigma_{0}$ is (a canonical parallel of) the attaching circle of $A$. Duals of the 2-handles in $M$ are attached to this level, and the disks $D_{*}^{ \pm}$go over these duals.

Thicken $\Sigma_{0} \cup D_{*}^{ \pm}$in the upper boundary of $W \cup(2$-handles of $M)$. This is the thickening as used in 3.2, minus a plug where $A$ passes through, so is $\left(S^{1} \times I\right) \times I$. The top of this is an annulus with one end $\partial A$ and going over duals of handles of $M$. The asymmetric description of the move given in 3.2.2 is to push the handles of $M$ off this annulus across $A^{*}$. The dual of this move is to move the attaching map of $A$ by isotopy along this annulus, going over duals of $M$-handles in the process. In particular the $W^{\prime}$ obtained at the end of the move is constructed by removing the handle $A$ from $W$ and reattaching it on the other end of the surface $\Sigma_{0}$.

This description shows that the link giving attaching maps in $W$ change by a variant of the "grope cobordism" of Conant and Teichner [CT]. Here the grope has height 1 (is just a surface), and the standard basis curves (attaching curves for caps) are required to be parallel to attaching maps of (duals of) handles of $M$. Grope cobordisms of greater height appear in the grope moves in 3.5.

3.4 Standard structure. The "standard structure" of a deformation (see the beginning of 2.2) is

(1) a canonical $\Gamma$ structure for the output decomposition, i.e. a factorization $\pi_{1} W^{\prime} \rightarrow \Gamma \rightarrow \pi_{1} N ;$ and

(2) a simple chain equivalence of chain complexes

$$
C_{*}\left(W, \partial_{0} W ; Z[\Gamma]\right) \rightarrow C_{*}\left(W^{\prime}, \partial_{0} W ; Z[\Gamma]\right) .
$$

A $(\Lambda, \Gamma)$ deformation has slightly more, namely

(3) a canonical refinement of the $\Gamma$-decomposition structure to a $\Lambda$ structure, i.e. the factorization above lifts canonically to $\pi_{1} W^{\prime} \rightarrow \Lambda$.

We now derive this structure.

Recall that a $\Gamma$ deformation is a sequence of decompositions, each obtained from the previous one by either ordinary handle moves in $M$ and $W$, or by a move described above. We begin with the ordinary handle moves. These do not change $M$ and $W$, so $\pi_{1} W$ does not change and the original $\pi_{1}$ factorization is used. The identity map $W \rightarrow W$ induces a chain map of complexes constructed using the two handle decompositions. But it is a standard fact that this is a simple chain equivalence. Indeed the definition of "simple chain equivalence" was developed exactly to encode the algebraic changes resulting from handle moves, so this chain map is simple essentially by definition.

It remains to describe structure for the new moves. On the $W$ side the move changes the attaching map of a handle $A$. The new attaching map of $A$ lifts to the $\Gamma$ cover, or $\Lambda$ cover in the $(\Lambda, \Gamma)$ case, so the homomorphism $\pi_{1}(W-A) \rightarrow \Gamma$ extends canonically to $\pi_{1} W^{\prime} \rightarrow \Gamma$ (respectively $\Lambda$ ). This provides the factoring. Next, there is an evident bijection of handles in $W$ and $W^{\prime}$. This bijection extends linearly to give based isomorphisms of the cellular chain groups $C_{*}\left(W, \partial_{0} W\right) \simeq$ $C_{*}\left(W^{\prime}, \partial_{0} W\right)$. On the group level this works with $\Lambda$ coefficients in the $(\Lambda, \Gamma)$ case. With $\Gamma$ coefficients this is an isomorphism of chain complexes, i.e. commutes with 
the boundary homomorphisms. This is clear for all generators except $A$, where it follows from the lifting of the surface $\Sigma_{0}$ as follows: Think of the chain groups as relative homology of skeleta,

$$
C_{i}\left(W, \partial_{0} W ; Z[\Gamma]\right)=H_{i}\left(W^{(i)}, W^{(i-1)} ; Z[\Gamma]\right) .
$$

Then $\partial A$ is the image of the attaching map in $H_{1}\left(W^{(1)}, W^{(0)} ; Z[\Gamma]\right)$. But the lift of $\Sigma_{0}$ to the $\Gamma$ cover provides a homology between the images of the two attaching maps, so they are equal in this group.

3.5 Grope moves. We first recall the definition of a grope (see FQ). A grope of height 1 is a capped surface, as described above. It has a standard embedding in $D^{3}$ with the boundary of the surface a standard circle in $S^{2}$. We now proceed recursively: suppose gropes of height $n-1$ are defined, and define gropes of height $n$ to be the result of replacing all the caps in a grope of height $n-1$ by capped surfaces. The simplest grope of height 2 is shown in Figure 4.

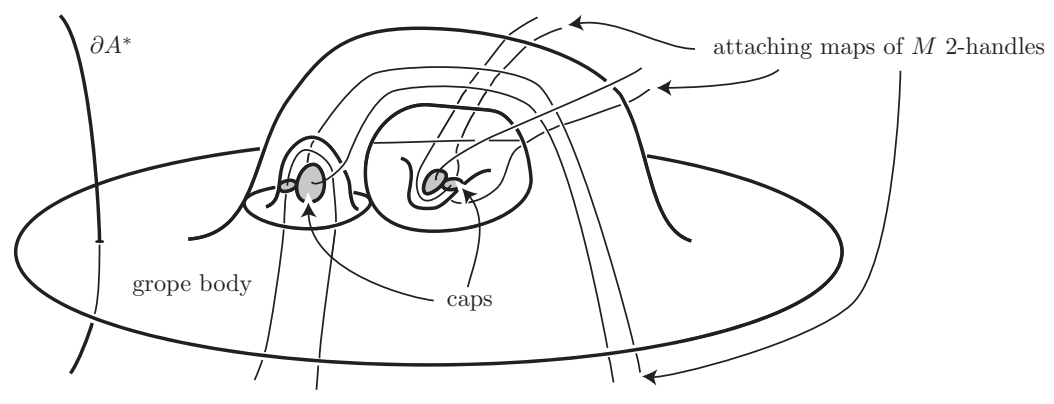

FiguRE 4.

The caps of the grope are the caps of the layer of capped surfaces. The rest of the grope is called the body, and consists of $n$ layers of surfaces. There is a standard model (embedding in $D^{3}$ ) obtained by starting with the standard model of the grope of height $n-1$ and replacing $D^{3}$ neighborhoods of its caps by standard models of capped surfaces. Further, these are all spines of $D^{3}$, or equivalently, the regular neighborhood of the grope is isomorphic to $D^{3}$. Embeddings and immersions of gropes are defined in terms of these standard neighborhoods: an embedding of a grope in a 4-manifold, for instance, is an embedding of $D^{3} \times I$, thought of as a neighborhood of the spine.

3.5.1 Data for a $\Gamma$ grope move. The data is the straightforward analog of 3.1:

(1) a capped grope of height $n$ embedded in $\partial_{1} M^{(1)}$;

(2) the body of the grope is disjoint from the attaching maps of the 2-handles of $M$, so lies in $\partial_{1} W$. This inclusion lifts to an inclusion of the body in the $\Gamma$ cover;

(3) the cap disks are disjoint from the 2-handles of $W$; and

(4) the body intersects (dual attaching maps of) 2-handles of $W$ in a single point in the lowest level surface.

This is illustrated in Figure 4. 
3.5.2 The move. The move itself is the straightforward elaboration of the height-1 case described in 3.2 and 3.3. Denote by $A^{*}$ the dual of the handle of $W$ that intersects the grope body, and arrange that its attaching map intersects the neighborhood $D^{3} \times I$ in an arc $\{x\} \times I$. The move pushes the $D^{3} \times I$ and all the stuff intersecting it across $A^{*}$, as in 3.2. Alternatively, it relocates the handle $A \subset W$ to one attached on the boundary circle of the grope body, as in 3.3.

3.6 Contraction, and standard structure for grope moves. The (one-fold) contraction of a grope undoes the last stage in the construction, which is to say it replaces the uppermost layer of capped surfaces by disks. This can be described explicitly in terms of the grope, by using some of the caps to do surgery on the uppermost surfaces, reducing them to disks. The $k$-fold contraction does this $k$ times. Thus the $k$-fold contraction of a grope of height $n$ gives a grope of height $n-k$, whose body is the first $n-k$ layers of surfaces in the original.

Contracting the grope in the data for a grope move gives data for a lower-height grope move. Geometrically the two moves have the same effect since the two gropes have the same regular neighborhood. They agree algebraically as well after taking into account a canonical improvement in the fundamental group structure. If $\pi_{1} W$ factors through $\Gamma$, then $\Gamma_{k}(W)$ is defined (Introduction and 2.3) to be $\pi_{1} W$ modulo the $k$-fold iterated commutator of the kernel of $\pi_{1} W \rightarrow \Gamma$.

Proposition. Suppose the body of a grope of height $n$ lifts to the $\Gamma$ cover of $W$. Then the union of the first $n-k$ layers lifts to the $\Gamma_{k}(W)$ cover.

Proof. Suppose we have an oriented surface with one boundary component. Then in $\pi_{1}$ the boundary curve is a product of commutators. More generally, the boundary curve of a grope of height $k$ is a $k$-fold iterated commutator in the fundamental group of the body. Now suppose $G$ is a capped grope of height $n$ with a map of the body to $W$ that lifts to the $\Gamma$ cover. This means the attaching curves for the caps lie in the kernel of $\pi_{1} W \rightarrow \Gamma$. The attaching curves on the body of the $k$-fold contraction are $k$-fold commutators of the original cap curves, so this body lifts to the $\Gamma_{k}(W)$ cover of $W$.

Setting $k=n-1$, and recalling that the standard moves are grope deformations of height 1 we get:

Corollary. A $\Gamma$ grope deformation of height $n$ is a $\Gamma_{n-1}(W)$-deformation.

There is a weak converse to this: suppose a grope body of height $n-k$ lifts to the $\Gamma_{k}(W)$ cover. Then it extends to a map of a grope body of height $n$ that lifts to the $\Gamma$ cover. In general this map will not be suitable for a deformation move because it is not an embedding. It may be possible to modify it to get an embedding; cf. I, $\S 7$, and 5.4 below.

3.7 Relation to finite type filtrations. "Finite type" invariants of links are defined to be ones that do not detect changes of sufficient complexity. Specifically a "degree $k$ " modification is defined as follows: suppose a 3-ball intersects the link in $k$ segments. Change the intersection with the ball to a new configuration with the property that if any one segment is omitted, then the result is isotopic (in the disk, rel boundary) to the corresponding omission from the original. An invariant is said to be of "finite type of degree $<k$ " if it is unchanged by degree- $k$ modifications. More precisely an invariant defined for a class of links is of finite type of degree $k$ if 
the class is closed under degree $k+1$ modifications and the invariant is unchanged by these modifications.

"Finite type" is related to the grope picture as follows. The simplest capped surface is a once-punctured torus with a single pair of caps. Similarly the simplest grope of height $n$ is obtained by always replacing caps with copies of this simplest capped surface. Figure 4 shows the simplest grope of height 2. Arbitrary grope moves can be subdivided into a sequence of moves all using the simplest grope, so we focus on these. Such a grope has $2^{n}$ caps. We can further suppose that the embeddings used as data for a move have each cap intersecting a single $M$ 2-handle in a point. The neighborhood of the grope is then a disk that intersects the attaching link of the 2-handles in $2^{n}$ segments. The grope move changes the attaching link by pushing it across $A^{*}$. But in fact we could pull all but one of these out of the disk, to arrange the intersection to be $2^{n}$ sub-segments of one of the original segments. After this the grope move changes only one of the original segments. Thus omitting any one of these segments trivializes the move. The conclusion is that grope moves of height $n$ on a link are modifications of degree $2^{n}$. Conant and Teichner [CT have developed a sharper relation between gropes and finite type that characterizes modifications of arbitrary degree.

This discussion shows that the solvable-tower version of chain invariants described in Section 6 are in an appropriate sense finite type invariants.

\section{Chain invariants}

In this section we define an invariant using equivalence classes of algebraic data coming from a $(\Lambda, \Gamma)$ deformation. This should be regarded as preliminary: even if it gives the right thing, the formulation needs refinement. See 4.6 for a possible relation to $K$-theory.

4.1 Small chain objects and $\operatorname{Sc}(Z[\Lambda]) \downarrow C$. We will say $D$ is a "small chain object over $Z[\Lambda]$ " if it satisfies the following:

(1) $D$ is a finitely generated free based $Z[\Lambda]$ chain complex;

(2) $D$ is homologically 2 -dimensional; and

(3) $H_{0}(D ; Z[\Lambda])$ is trivial as a $Z[\Lambda]$ module.

Small chain objects encode the algebraic properties of chains of a 2-complex, or relative chains of a relative 2-complex. In particular if $M \cup W$ is a $\Lambda$ decomposition, then the cellular chain complex $C_{*}\left(W, \partial_{0} W ; Z[\Lambda]\right)$ is a small chain object.

Denote by $\operatorname{Sc}(Z[\Lambda])$ the category of small chain objects and simple chain equivalences. A homomorphism $\Lambda \rightarrow \Gamma$ induces a functor of categories by $D \mapsto D \otimes Z[\Gamma]$.

Now suppose $C$ is a small chain object over $Z[\Gamma]$. Then we denote the fiber over $C$ of the functor $\operatorname{Sc}(Z[\Lambda]) \rightarrow \operatorname{Sc}(Z[\Gamma])$ by $\operatorname{Sc}(Z[\Lambda]) \downarrow C$. Explicitly this is the category with objects $(D, f)$, where $D$ is a small chain object over $Z[\Lambda]$ and $f$ is a simple chain equivalence $D \otimes Z[\Gamma] \rightarrow C$. Morphisms are simple chain equivalences over $Z[\Lambda]$ whose $Z[\Gamma]$ reductions chain homotopy commute with the given equivalences.

The morphisms define an equivalence relation on objects of $\operatorname{Sc}(Z[\Lambda]) \downarrow C$. We abuse the notation by using $\operatorname{Sc}(Z[\Lambda]) \downarrow C$ to also refer to the set of equivalence classes. The basic plan is to use the equivalence class of the chain complex of $W$ as our invariant. The definition above is sufficient when $\partial_{0} W$ is empty, but has to be elaborated a bit in general. This is done in the next two sections. 
4.2 Chains with stratified coefficients. When $\partial_{0} W$ is nonempty we need stratified coefficients to record $\pi_{1}$ information on the boundary. In fact we use the language "stratified coefficients", but only describe the very special case needed rather than develop a general theory and specialize it.

The "suspension" of a complex is defined by shifting the groups down one degree and multiplying the boundary homomorphisms by $(-1)^{n}$. In detail the suspension of $C_{*}$ is denoted by $C_{*-1}$. The $n^{\text {th }}$ chain group of $C_{*-1}$ is $C_{n-1}$, and the boundary from degree $n$ to degree $n-1$ is $(-1)^{n-1} \partial: C_{n-1} \rightarrow C_{n-2}$.

If $(X, Y)$ is a CW pair, then there is a boundary chain map of cellular chain complexes $d: C_{*}(X, Y) \rightarrow C_{*-1}(Y)$ that on homology induces the boundary homomorphism in the long exact sequence. Here the target is the suspension as defined above, and the signs on the boundary homomorphisms are needed to make this a chain map.

Now suppose $\lambda: \Lambda \rightarrow \Gamma$ is a homomorphism of groups, $(X, Y)$ is a CW pair, and there is a commutative diagram:

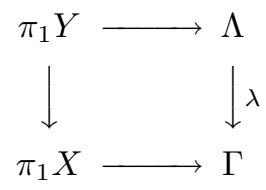

Then we define the stratified chain complex $C_{*}(X, Y ; Z[\Lambda])$ to be the pullback of the diagram:

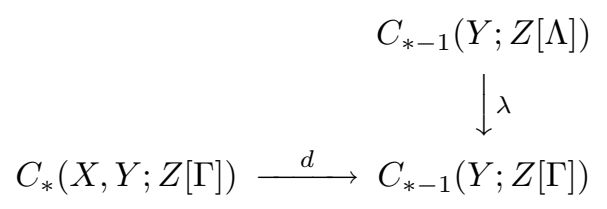

Explicitly the $n^{\text {th }}$ chain group in the pullback is given by $(x, y) \in C_{n}(X, Y ; Z[\Gamma]) \oplus$ $C_{n-1}(Y ; Z[\Lambda])$ such that $d(x)=\lambda(y)$, where $\lambda$ denotes a change-of-coefficient map on chains induced by the homomorphism $\lambda$.

The facts we need about this complex are:

(1) $C_{*}(X, Y ; Z[\lambda])$ is a chain complex over $Z[\Lambda]$, though the chain groups are not free;

(2) $C_{*}(X, Y ; Z[\lambda]) \otimes Z[\Gamma]$ is $C_{*}(X, Y ; Z[\Gamma])$, so is free and based over $Z[\Gamma]$; and

(3) if the homomorphism $\pi_{1} X \rightarrow \Gamma$ lifts to $\Lambda$ compatibly with the homomorphism on $\pi_{1} Y$, then there is a natural $Z[\Lambda]$ chain map $C_{*}(X, Y ; Z[\Lambda]) \rightarrow$ $C_{*}(X, Y ; Z[\lambda])$ induced by the universal property of the pullback and the commutative diagram:

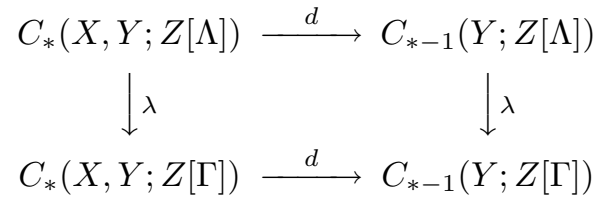

4.3 Small chain objects over stratified chains. The definition of the category $\operatorname{Sc}(Z[\Lambda]) \downarrow C$ given in 4.1 requires $C$ to be a small chain object over $Z[\Gamma]$. Here we extend this slightly to include stratified chains. Suppose $C$ is

(1) a chain complex (not necessarily free) over $Z[\Lambda]$, and

(2) $C \otimes Z[\Gamma]$ is a small chain object over $Z[\Gamma]$. 
Then we define $\operatorname{Sc}(Z[\Lambda]) \downarrow C$ to be the category with objects $(E, f)$, where $E$ is a small chain object over $Z[\Lambda]$ and $f: E \rightarrow C$ is a $Z[\Lambda]$ chain map whose $Z[\Gamma]$ reduction is a simple equivalence. Morphisms in the category are simple equivalences over $Z[\Lambda]$ that chain homotopy commute with the maps to $C$.

As before we use the same notation for the set of equivalence classes of objects.

4.4 Proposition (Chain invariant realization). Suppose $\Lambda \stackrel{\lambda}{\longrightarrow} \Gamma \rightarrow \pi_{1} N$ are given and $N=M \cup W$ is a $\Lambda$-decomposition. Then the stratified chains $C=$ $C_{*}\left(W, \partial_{0} W ; Z[\lambda]\right)$ are defined. If $f: M \cup W \mapsto M^{\prime} \cup W^{\prime}$ is a $\Gamma$ deformation so that the lift on $\pi_{1} \partial_{0} W$ extends to $\pi_{1} W^{\prime} \rightarrow \Lambda$, then $\left(C_{*}\left(W^{\prime}, \partial_{0} W ; Z[\Lambda]\right), f_{*}\right)$ represents a class in $\operatorname{Sc}(Z[\Lambda]) \downarrow C$. Conversely any such class is realized by some deformation and lift.

Refinement. If $\Lambda \rightarrow \Gamma$ factors through $\Gamma_{n}(W)$, then invariants can be realized by $\Gamma$ grope deformation of height $n+1$.

Proof. Putting together the $H_{0}$ hypothesis on small chain objects, the chain equivalence, and the fact that $M \cup W$ is a dual decomposition, we see the natural maps induce isomorphisms

$$
H_{0}(D ; Z[\Lambda]) \simeq H_{0}(D ; Z[\Gamma]) \simeq H_{0}\left(W, \partial_{0} W ; Z[\Gamma]\right) \simeq H_{0}\left(N, \partial_{0} W ; Z\left[\pi_{1} N\right]\right) .
$$

This means, according to the Chain Realization Theorem for $\Lambda$, there is a $\Lambda$ decomposition $\widehat{W} \cup \widehat{M}$ with a simple chain equivalence $D_{*} \stackrel{\sim}{\longrightarrow} C_{*}\left(\widehat{W}, \partial_{0} W ; Z[\Lambda]\right)$.

Let $\left(K, \partial_{0} W\right) \rightarrow\left(N, \partial_{0} W\right)$ be the spine of $\widehat{W}$. Then $K$ and the chain equivalence over $\Gamma$ satisfy the hypotheses of the Spine Realization Theorem. Thus there is a $\Gamma$ deformation of $M \cup W$ to a decomposition $M^{\prime} \cup W^{\prime}$ and a homotopy deformation of the the spine of $W^{\prime}$ to $K$. Further these realize the input data in the sense that the canonical chain equivalence from the $\Gamma$ deformation is chain homotopic to the input equivalence. We conclude that the decomposition $M^{\prime} \cup W^{\prime}$ and its associated data does realize the given chain invariant.

The refinement is obtained by substituting 5.3 for 5.2 in this argument.

4.5 $(\Lambda, \Gamma)$ deformation. The $\pi_{1} W^{\prime}$ lift to $\Lambda$ needed to define a chain invariant comes automatically in a $(\Lambda, \Gamma)$ deformation. A natural question is therefore: are all chain invariants realized by $(\Lambda, \Gamma)$ deformation? Proposition 4.4 does not show this, and we suspect that there is significant structure not identified in 4.3 that limits the classes realized. For instance the $(\Lambda, \Gamma)$ deformation moves come with a canonical basis-preserving isomorphism of the $Z[\Lambda]$ chain groups. This is usually not a chain map, but it may be close enough to one to show that some variant of Whitehead torsion vanishes. A possible such variant is described below. Or a $(\Lambda, \Gamma)$ deformation may have a "trace" embedded in $N \times I$ that gives a concordance between the two decompositions. In that case a bilinear invariant would be defined, and the linear invariants would be limited to the image of the bilinear ones.

4.6 $K$-theory. Suppose $\Lambda \rightarrow \Gamma$ is a homomorphism and $f: D \rightarrow E$ is a chain map of $Z[\Lambda]$ complexes that becomes a simple chain equivalence when tensored with $Z[\Gamma]$. Then it is also a chain equivalence over the Cohn localization $Z[\Lambda]_{\Sigma}$, obtained by inverting the set $\Sigma$ of square matrices over $Z[\Lambda]$ whose reductions to $Z[\Gamma]$ are isomorphisms. See Ranicki $[\mathbf{R}]$ for an overview of topological applications of Cohn localizations. The chain equivalence has a torsion $\tau(f) \in K_{1}\left(Z[\Lambda]_{\Sigma}\right) / \pm \Lambda$. Here, as usual in the Whitehead group, we divide out the units $\pm \Lambda$ to make the 
torsion independent of choice of basis cells in the $\Lambda$ cover. Since $f$ is simple over $Z[\Gamma]$, this torsion lies in the kernel of the homomorphism to $K_{1}(Z[\Gamma]) / \pm \Gamma$.

4.6.1 Question. Does a $(\Lambda, \Gamma)$ deformation define a torsion in

$$
\operatorname{ker}\left(K_{1}\left(Z[\Lambda]_{\Sigma}\right) \rightarrow K_{1}(Z[\Gamma])\right) / \pm \Lambda ?
$$

For the next question we suppose $h: C \rightarrow D$ is a $Z[\Lambda]$ chain map, $C$ is a small chain object over $Z[\Lambda], D \otimes Z[\Gamma]$ is a small chain object, and $h \otimes Z[\Gamma]$ is a simple equivalence. We are thinking of the $Z[\Lambda]$ chains mapping to the stratified chains of a pair $\left(W, \partial_{0} W\right)$. Then there is a natural map $\Theta_{h}$,

$$
\operatorname{ker}\left(K_{1}\left(Z[\Lambda]_{\Sigma}\right) \rightarrow K_{1}(Z[\Gamma])\right) / \pm \Lambda \stackrel{\Theta_{h}}{\longrightarrow} \operatorname{Sc}(Z[\Lambda]) \downarrow D,
$$

defined by composing $\partial: C_{2} \rightarrow C_{1}$ with a homomorphism $C_{2} \rightarrow C_{2}$ representing the $K$-theory class. We expect that in general the chain invariant group depends on $C$ and $D$ and not just $\Lambda \rightarrow \Gamma$. However there may be useful cases in which they agree:

4.6.2 Question. Is $\Theta_{h}$ an isomorphism if either

(1) $C_{i}=0$ for $i \neq 2$, or

(2) $\Lambda=\Gamma_{n}(W)$ and $C \rightarrow D$ are the $\Lambda$ and stratified chains for some $\Gamma$ decomposition $M \cup W$.

The difference between the two groups concerns whether simple chain equivalences over $Z[\Gamma]$ can be lifted in a reasonably canonical way to $Z[\Lambda]$ chain maps. The idea in the first case is that the model is so small that not too much can go wrong. The reason this case is of interest is that it may include decompositions manufactured to study more general links. In the second case the idea is that behavior in the chain complexes in degrees less than 2 is determined by the group, so again problems are concentrated in degree 2. This case is interesting because it applies to the solvable tower analysis.

\section{Relative versions, AND PROOFs of REAlization}

In this section we state relative versions of the theorems in 2.1-2.3 and give proofs. The proofs are mostly minor modifications of the proofs of Part I.

As in Section 3 we fix a compact smooth 4-manifold $N$ and a decomposition of its boundary into $\partial N=\partial_{0} M \cup \partial_{0} W$. Again, $M$ and $W$ are not yet defined; the notations for the pieces of $\partial N$ are for later convenience. We also fix a homomorphism $\gamma: \Gamma \rightarrow \pi_{1} N$ and a factoring $\pi_{1} \partial_{0} W \rightarrow \Gamma \rightarrow \pi_{1} N$, and consider $\Gamma$-decompositions $N=M \cup W$ that extend the structure on $\partial N$.

The first topic is the extension of the chain realization theorem 2.1. Note that the factoring of $\pi_{1} \partial_{0} W$ through $\gamma: \Gamma \rightarrow \pi_{1} N$ means that the stratified chains $C_{*}\left(N, \partial_{0} W ; Z[\gamma]\right)$ are defined. Recall that a "small chain object" is a free based homologically 2-dimensional complex with $H_{0}$ trivial as a $Z[\Gamma]$ module.

5.1 Theorem (Relative $\Gamma$-Chain Realization). A $\Gamma$ decomposition $N=M \cup W$ defines a small chain object $D=C_{*}\left(W, \partial_{0} W ; Z[\Gamma]\right)$ over $Z[\Gamma]$ and a $Z[\Gamma]$ chain map $f: D \rightarrow C_{*}\left(N, \partial_{0} W ; Z[\Gamma]\right)$ whose $Z\left[\pi_{1} N\right]$ reduction is an isomorphism on $H_{0}$ and an epimorphism on $H_{1}$. Conversely given such $D$ and $f$ there is a $\Gamma$ decomposition that realizes it up to simple chain homotopy. 
The first step is to modify the 1-skeleton alignment lemma of Part I, §4. The modifications are relatively minor, but the statement is complicated enough that we repeat the whole thing to be safe.

5.2 1-Skeleton Alignment Lemma. Suppose $\Gamma \rightarrow \pi, C_{*}$ is a free based $Z[\Gamma]$ complex whose $H_{0}$ is trivial as a $Z[\Gamma]$ module, $D_{*}$ is a free based $Z[\pi]$ complex, and $f: C_{*} \rightarrow D_{*}$ is a $Z[\Gamma]$ chain map whose $Z[\pi]$ reduction is an isomorphism on $H_{0}$ and an epimorphism on $H_{1}$. Then there is a chain homotopy commutative diagram

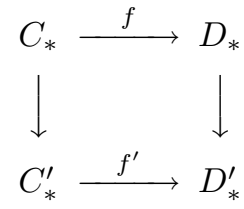

so the vertical maps are simple equivalences over $Z[\Gamma]$ and $Z[\pi]$ respectively and are isomorphisms in degrees $\geq 3$, and $f^{\prime}$ is basis-preserving in degrees 0 and 1 . Further:

If $D_{*}$ is the cellular chains of a CW complex or pair (resp. 4-d handlebody) with connected $\Gamma$ cover, then $D_{*} \rightarrow D_{*}^{\prime}$ can be arranged to be induced by a homotopy 2-deformation (resp. handlebody moves).

If both $C_{*}$ and $D_{*}$ are cellular chains of $C W$ complexes or pairs (resp. 4-d handlebodies) with connected covers and the isomorphism on $H_{0}$ is the identity, then both $C_{*} \rightarrow C_{*}^{\prime}$ and $D_{*} \rightarrow D_{*}$ can be arranged to be induced by 2-deformation (resp. handle moves).

To make sense of the requirement that $f$ be a $Z[\Gamma]$ chain map we regard $D_{*}$ as a $Z[\Gamma]$ complex via the module structures induced by the ring map $Z[\Gamma] \rightarrow Z[\pi]$. Such a chain map factors through the quotient $C_{*} \rightarrow C_{*} \otimes Z[\pi]$ and a $Z[\pi]$ chain map $C_{*} \otimes Z[\pi] \rightarrow D_{*}$. This latter chain map is the " $Z[\pi]$ reduction" of $f$.

Proof. The proof is obtained by modifying the proof of I, $\S 4$. In the geometric cases the new hypothesis are automatically satisfied, and the new conclusion follows for free because cell arguments lift to any cover. The cases that require modification are "semi-algebraic 0-skeleton alignment" and "algebraic 1-skeleton alignment."

In the semi-algebraic 0-skeleton argument a copy of $D_{0}$ is added to $C_{0}$. In order to get a free $\Gamma$ complex we use a free $Z[\Gamma]$ module $\hat{D}_{0}$ with the same basis as $D_{0}$. Next we must lift $f_{0}$ and $g_{0}$ to homomorphisms $\hat{f}_{0}$ and $\hat{g}_{0}$ with $Z[\Gamma]$ coefficients, so that equation (2) in I, $\S 4$,

$$
\hat{f}_{0} \hat{g}_{0}+\partial t=\mathrm{id}_{C}
$$

holds with $Z[\Gamma]$ coefficients. Previously it was done with $Z[\pi]$ coefficients. To get the improvement we need that the change-of-coefficient homomorphism $C \rightarrow$ $C \otimes Z[\pi]$ induces an isomorphism on $H_{0}$. But this follows from the hypothesis that $H_{0}$ is trivial as a $Z[\Gamma]$ module. After this adjustment the argument proceeds as before.

In the algebraic 1-skeleton alignment we take $\hat{D}_{1}$ a free $Z[\Gamma]$ module whose $Z[\pi]$ reduction is $D_{1}$. Lift $g: D_{1} \rightarrow C_{1} \otimes Z[\pi]$ to $\hat{g}: \hat{D}_{1} \rightarrow C_{1}$. Then the two right columns in the $3 \times 3$ diagram below equation (1) in I, $\S 4$, become simple equivalences of $Z[\Gamma]$ complexes. The lower vertical arrow in the second column becomes $(\partial, \partial \hat{g})$. This does not affect the rest of the argument since the $Z[\pi]$ reduction of $\partial \hat{g}$ can be identified with $\partial$, as was done in the original argument.

This ends the modification of the proof of the 1-skeleton alignment lemma. 
Proof of Theorem 5.1. The rest of the proof of Theorem 5.1 is obtained by routine modifications of Part I, $\S 5$. These are even more minor than the modifications needed in 1-skeleton alignment, so are omitted.

Next is the bounded version of spine realization. Again we describe data coming from a deformation, and the theorem asserts that any such data is realized. The main difference is the appearence of stratified chains encoding the fixed structure on the boundary. Suppose $d: M \cup W \mapsto M^{\prime} \cup W^{\prime}$ is a $\Gamma$ deformation, and denote the (relative) spine of $W^{\prime}$ by $\left(K, \partial_{0} W\right)$. Then

(1) $\left(K, \partial_{0} W\right) \rightarrow N$ is a relative 2-complex and the map is the identity on $\partial_{0} W$;

(2) there is a factoring of $\pi_{1} K$ through $\Gamma$ that extends the given factoring on $\pi_{1} \partial_{0} W ;$ and

(3) the deformation induces a simple chain equivalence $d_{*}: C_{*}\left(K, \partial_{0} W ; Z[\Gamma]\right)$ $\rightarrow C_{*}\left(W, \partial_{0} W ; Z[\Gamma]\right)$ that chain homotopy commutes with the maps to the stratified chains $C_{*}\left(N, \partial_{0} W ; Z[\gamma]\right)$.

5.3 Theorem (Relative $\Gamma$-Spine Realization). Suppose $N=M \cup W$ is a $\Gamma$ decomposition, $\left(K, \partial_{0} W\right) \rightarrow\left(N, \partial_{0} W\right)$ is a relative 2 -complex, and

$$
d_{*}: C_{*}\left(K, \partial_{0} W ; Z[\Gamma]\right) \rightarrow C_{*}\left(W, \partial_{0} W ; Z[\Gamma]\right)
$$

is a chain map satisfying the conditions above. Then there is a $\Gamma$ deformation of $M$ to a decomposition $M^{\prime} \cup W^{\prime}$ so that the spine of $\left(W^{\prime}, \partial_{0} W\right)$ realizes $\left(K, \partial_{0} W\right)$ up to homotopy deformation.

As in 2.2 the homotopy deformation can be arranged to realize the chain data.

Proof. The proof closely follows the proof in Part I, $\S 7$. Note, however, that $M$ and $W$ are interchanged in the notation used here and in I, $\S 7$.

5.3.1 Align 1-skeleta. Use the improved 1-skeleton alignment lemma above to change the handle structure on $\left(W, \partial_{0} W\right)$ and homotopy deform $\left(K, \partial_{0} W\right)$ so the 1-skeleta can be identified. Specifically we get

(1) $K=W^{(1)} \cup(2$-cells);

(2) the homomorphisms $\pi_{1} K \rightarrow \Gamma$ and $\pi_{1} W \rightarrow \Gamma$ agree on the 1-skeleton;

(3) the simple chain equivalence $d_{*}: C_{*}\left(K, \partial_{0} W ; Z[\Gamma]\right) \rightarrow C_{*}\left(W, \partial_{0} W ; Z[\Gamma]\right)$ is the identity on $C_{0}$ and $C_{1}$, and is a basis-preserving isomorphism on $C_{2}$; and

(4) the chain maps to the stratified chains $C_{*}\left(N \partial_{0} W ; Z[\gamma]\right)$ agree on the 0 - and 1-chains, and commute with $d_{*}$.

Note that $\Gamma$-deformations (of $M$ ) preserve all this data. Deformations do not change the 1-skeleton of $W$, so do not affect (1) and (2). The 2-handles do change, but the standard data gives a basis-preserving chain map of $Z[\Gamma]$ chains from the old to the new, so composing this with the chain equivalence (3) replicates this chain data. Finally the standard data chain map is the identity on the 1-skeleton, so condition (4) is not disturbed either.

5.3.2 2-cell data. To work with 2-cells we need the absolute rather than the relative chain complexes. It is this step that requires use of stratified chains. The relative chains of both $K$ and $W$ map to the stratified chains of $\left(N, \partial_{0} W\right)$, and this in turn 
has a boundary map to the $Z[\Gamma]$ chains of $\partial_{0} W$. This defines the right-hand square in the diagram

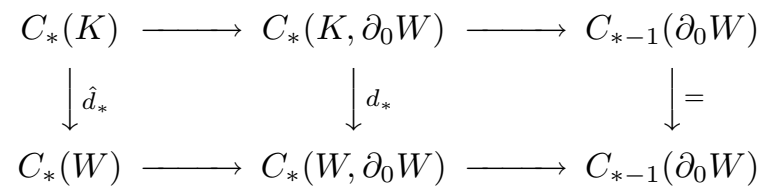

where all coefficients are $Z[\Gamma]$. The right-hand square commutes, and so induces the left vertical chain map $\hat{d}_{*}$. This is also the identity on the 1-skeleton and a basis-preserving isomorphism on the 2-cells.

The chain map $\hat{d}_{*}$ gives $Z[\Gamma]$ homologies between attaching maps of the 2-handles of $W$ and the 2-cells of $K$. To see these recall that the cellular chain complex is defined to have chain groups $C_{i}(K)=H_{i}\left(K^{(i)}, K^{(i-1)}\right)$, where $K^{(i)}$ denotes the $i$-skeleton, and boundaries are defined by compositions

$$
H_{i}\left(K^{(i)}, K^{(i-1)}\right) \stackrel{\partial}{\longrightarrow} H_{i-1}\left(K^{(i-1)}\right) \rightarrow H_{i-1}\left(K^{(i-1)}, K^{(i-2)}\right) .
$$

Since $d_{2}$ takes 2-cells to 2 -handles, their boundaries must be equal in $H_{1}$ of the 1-skeleton.

We claim that to prove the theorem it is sufficient to deform $W$ so these $Z[\Gamma]$ homologies are realized by homotopies. First, the homotopies give the desired 2-deformation between $K$ and the spine of $W$. However we also want this 2deformation to homotopy commute with the maps to $N$, and for this we need to extend the homotopies of attaching maps to homotopies between the maps of the 2-cells into $N$. The obstruction to finding such a homotopy is an element of $\pi_{2} N$ formed from a 2-cell in each of $K$ and $W$, and the homotopy between the attaching maps. Since we are working in the universal cover of $N, \pi_{2} N=H_{2}\left(N ; Z\left[\pi_{1} N\right]\right)$ and it is sufficient to show the homology class vanishes.

The inclusion $\left(W, \partial_{0} W\right) \rightarrow\left(N, \partial_{0} W\right)$ induces a commutative diagram

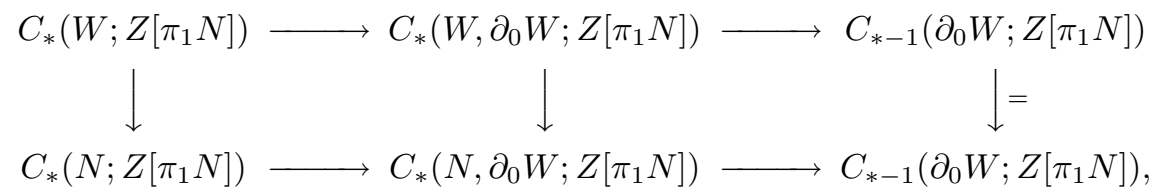

and similarly for $\left(K, \partial_{0} W\right) \rightarrow\left(N, \partial_{0} W\right)$. The complex $C_{*}\left(N, \partial_{0} W ; Z\left[\pi_{1} N\right]\right)$ is the $Z\left[\pi_{1} N\right]$ reduction of the stratified chains, so the commutativity hypothesis on the maps to stratified chains shows these inclusion diagrams commute with the chain maps $d_{*}$ and $\hat{d}_{*}$ described above. Now we again use the fact that $\hat{d}_{2}$ preserves bases. This and the commutativity mean a 2-cell of $K$ and the corresponding 2-handle of $W$ have the same image in $C_{2}\left(N ; Z\left[\pi_{1} N\right]\right)$. This in turn implies that the difference between them represents the trivial class in $H_{2}\left(N ; Z\left[\pi_{1} N\right]\right)$, as required.

5.3.3 Deform 2-cells. The situation is that we have $Z[\Gamma]$ homologies (in the common 1-skeleton) between attaching maps of 2-cells of $K$ and 2-handles of $W$, and we want to $\Gamma$-deform $W$ to $W^{\prime}$ so the corresponding homologies are realized by homotopies. Recall again that $\Gamma$-deformations do not change any of the skeleton or chain data. Also note that a single deformation move changes only one 2-handle, so it is sufficient to improve 2-handles one at a time.

The remainder of the proof is the same as the proof in $\S 7$ of Part I. In brief we represent the homology by a map of a surface into the 1-skeleton. Since we are 
using (at least) $Z\left[\pi_{1}\right]$ coefficients, this surface factors through the universal cover of $N$. This means it extends to a map of a capped surface into $N$. We manipulate this capped surface until we get the geometric data of Section 3 for a deformation move. The fact that the surface comes from $Z[\Gamma]$ homology means that it actually factors through the $\Gamma$ cover, but this plays no role in the construction until the very end when it is used to recognize the move as a $\Gamma$ deformation.

We warn again that in the proof of $\S 7$ of Part I, the notations $M$ and $W$ are interchanged from their meanings here.

Finally we have the grope refinement, extending 2.3.

5.4 Theorem (Relative $\Gamma_{n}$-Spine Realization). Suppose $N=M \cup W$ is a $\Gamma$ decomposition and $\left(K, \partial_{0} W\right) \rightarrow\left(N, \partial_{0} W\right)$ is a relative 2 -complex as in 5.3 , but with a factorization $\pi_{1} K \rightarrow \Gamma_{n}(W) \rightarrow \pi_{1} N$. Then there is a $\Gamma$ grope deformation of height $n+1$ from $M$ to a $\Gamma$ decomposition $M^{\prime} \cup W^{\prime}$ so that the spine of $\left(W^{\prime}, \partial_{0} W\right)$ realizes $\left(K, \partial_{0} W\right)$ with its $\Gamma_{n}$ factorization, if and only if the chain hypotheses of 5.3 hold over $\Gamma_{n}(W)$.

Proof. Follow the proof of 5.3, replacing $\Gamma$ by $\Gamma_{n}(W)$, up to the point in 5.3 .3 , where the homology data is used to get a surface in the $\Gamma_{n}(W)$ cover of the 1-skeleton giving a homology between attaching maps. Choose standard basis curves for the homology of this surface, but now observe that the lift to $\Gamma_{n}(W)$ implies that these curves bound uncapped gropes of height $n$ mapping into the $\Gamma$ cover of $W$. Adding these to the original surface gives an uncapped grope of height $n+1$. As before the factorization through $\Gamma \rightarrow \pi_{1} N$ implies that the uncapped grope in $W$ extends to a map of a capped grope into $N$.

From here the proof again essentially follows Part I, $\S 7$ : we see that by changing the attaching maps in $K$ by homotopy we can reduce the singularities in the map of the capped grope until we get embedded data as in 3.5, and can then do a grope deformation move on a handle of $W$. The surface and grope cases are not essentially different, so we will not give details. See Conant and Teichner [CT] for similar arguments for desingularizing maps of gropes.

\section{The SOlVABle TOWER}

The chain invariant of Section 4 is intended to be used to distinguish different decompositions of a 4-manifold. There is a difficulty in that before the invariant is defined we need to know there are some similarities, particularly in fundamental groups. The "solvable tower" is based on the fact that some fundamental group similarities can be detected homologically because the abelianization of $\pi_{1}$ is $H_{1}$. The outcome is an "obstruction theory": a sequence of invariants so that at each level either the invariant distinguishes the decompositions, or the next level invariant is defined.

As before we fix a smooth compact 4-manifold $N$, a homomorphism $\gamma: \Gamma \rightarrow$ $\pi_{1} N$, and a decomposition $\partial N=\partial_{0} M \cup \partial_{0} W$ with a factorization $\pi_{1} \partial_{0} W \rightarrow \Gamma \rightarrow$ $\pi_{1} N$. We will be considering derived groups $\Gamma_{n}(W)$, so nontrivial examples may arise even if we start with $\Gamma=\pi_{1} N$.

6.1 Homological equivalence. Suppose $M \cup W$ and $M^{\prime} \cup W^{\prime}$ are $\Gamma$ decompositions of $N$. We define a homological $\Gamma_{n}$ equivalence between them to be $(\phi, f)$, 
where

(1) $\phi$ is an isomorphism $\Gamma_{n}(W) \rightarrow \Gamma_{n}\left(W^{\prime}\right)$ that commutes with the homomorphisms from $\pi_{1} \partial_{0} W$ and to $\Gamma$; and

(2) $f$ is a simple chain homotopy equivalence of $Z\left[\Gamma_{n}\right]$ complexes,

$$
C_{*}\left(W, \partial_{0} W ; Z\left[\Gamma_{n}\right]\right) \stackrel{f}{\longrightarrow} C_{*}\left(W, \partial_{0} W ; Z\left[\Gamma_{n}\right]\right)
$$

that chain homotopy commutes with the maps to the stratified chains $C_{*}\left(N, \partial_{0} W ; Z\left[\gamma_{n}\right]\right)$.

In (2) we are using the isomorphism $\phi$ to identify the $\Gamma_{n}$ groups, and $\gamma_{n}: \Gamma_{n} \rightarrow$ $\pi_{1} N$ is the natural map.

6.2 Lemma. Suppose $(\phi, f)$ is a $\Gamma_{n}$ homological equivalence of decompositions. Then $\phi$ lifts to a canonical isomorphism $\hat{\phi}: \Gamma_{n+1}(W) \rightarrow \Gamma_{n+1}\left(W^{\prime}\right)$ and $f$ lifts to a $Z\left[\Gamma_{n+1}\right]$ equivalence $\hat{f}: C_{*}\left(W, \partial_{0} W ; Z\left[\gamma_{n+1, n}\right]\right) \rightarrow C_{*}\left(W^{\prime}, \partial_{0} W ; Z\left[\gamma_{n+1, n}\right]\right)$ of stratified chains.

The coefficient homomorphism in the stratified chain groups is the quotient $\gamma_{n+1, n}: \Gamma_{n+1}(W) \rightarrow \Gamma_{n}(W)$.

Proof. It is sufficient to do this for $\Gamma_{1}$, since $\Gamma_{n+1}=\left(\Gamma_{n}\right)_{1}$, and the notation is easier. In this case $\phi$ is the identity $\Gamma \rightarrow \Gamma$, and $f$ is a simple equivalence of $Z[\Gamma]$ chains.

The basic idea is that the kernel of $\Gamma_{1}(W) \rightarrow \Gamma$ is $H_{1}(W ; Z[\Gamma])$. If $W$ and $W^{\prime}$ have the same $\Gamma$, and the same $H_{1}$ by homological equivalence, then they should have the same $\Gamma_{1}$. There are two issues to deal with: in the relative case we get a relative $H_{1}$ rather than the absolute one needed, and there is a group extension problem.

By hypothesis the chain map $f$ homotopy commutes with the maps to the stratified chains $C_{*}\left(N, \partial_{0} W ; Z[\gamma]\right)$. The compositions with the boundary map to $C_{*-1}\left(\partial_{0} W ; Z[\Gamma]\right)$ therefore also commute. This shows that the right-hand square in the diagram below homotopy commutes, and since the rows are exact this induces the chain map $g$ :

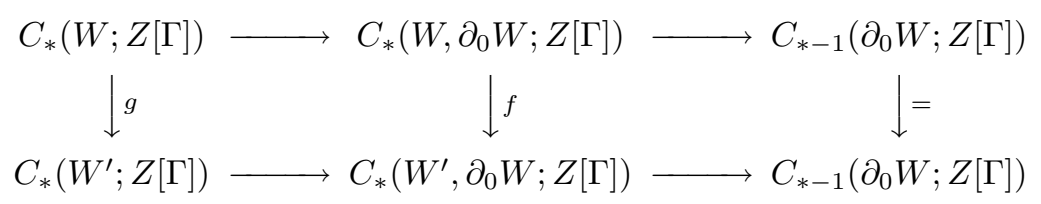

The chain map $g$ is a simple equivalence since the other two vertical maps are also. According to the 1-skeleton alignment lemma we can deform the spines of $W$ and $W^{\prime}$ to have the same 1-skeleton. Denote the fundamental group of this 1-skeleton by $F$. The kernel of $F \rightarrow \Gamma$ is the fundamental group of 1-skeleta for both $\Gamma$ covers; denote this kernel by $K$. By abelianization $K$ maps to $H_{1}(W, Z[\Gamma])$ and $H_{1}\left(W^{\prime}, Z[\Gamma]\right)$. The chain equivalence gives an isomorphism of these groups, and since the chain map is the identity in degree 1 the maps $K \rightarrow H_{1}$ commute with this isomorphism. This means their kernels are the same. But $\Gamma_{1}(W)=F / \operatorname{ker}\left(K \rightarrow H_{1}(W ; Z[\Gamma])\right)$ and similarly for $\Gamma_{1}\left(W^{\prime}\right)$. Therefore the kernels being the same gives an identification $\hat{\phi}: \Gamma_{1}(W)=\Gamma_{1}\left(W^{\prime}\right)$. 
To complete the lemma we must lift $f$ to a chain equivalence of stratified chains. The stratified chains of $W$ are defined to be the pullback in the diagram

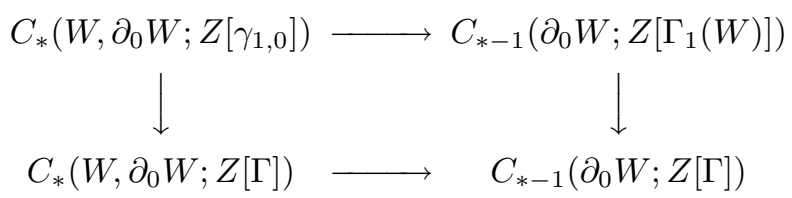

and similarly for $W^{\prime}$. To get a chain equivalence of stratified chains it is sufficient to get an equivalence of pullback data for $W$ and $W^{\prime}$. On the lower left we have the chain equivalence $f$. On the lower right we have the identity, or more precisely the equivalence induced by the identity on $\partial_{0} W$ and the isomorphism $\phi: \Gamma=\Gamma$. On the upper right we have a similar equivalence, induced by the identity on $\partial_{0} W$ and the new isomorphism $\hat{\phi}: \Gamma_{1}(W) \rightarrow \Gamma_{1}\left(W^{\prime}\right)$. These are compatible, so by universality of the pullback construction they define a chain equivalence of stratified chains.

6.3 The chain invariant of a homological equivalence. Suppose $(\phi, f)$ is a homological $\Gamma_{n}$ equivalence from $M \cup W$ to $M^{\prime} \cup W^{\prime}$. Then the chain invariant $\tau_{n+1}(\phi, f)$ is defined to be the equivalence class of $\left(C_{*}\left(W^{\prime}, \partial_{0} W ; Z\left[\Gamma_{n+1}\right]\right), q\right)$ in $\operatorname{Sc}\left(Z\left[\Gamma_{n+1}\right]\right) \downarrow C_{*}\left(W, \partial_{0} W ; Z\left[\gamma_{n+1, n}\right]\right)$.

Here $\Gamma_{i}$ refers to either $W$ or $W^{\prime}$, with the two groups being identified via $\phi$ when $i=n$, and by the lift $\hat{\phi}$ of 6.2 when $i=n+1 . \gamma_{n+1, n}$ is the natural quotient $\Gamma_{n+1} \rightarrow \Gamma_{n}$, and $q$ is the $Z\left[\Gamma_{n+1}\right]$ chain map obtained by composing the change-ofcoefficient map with the equivalence of 6.2 :

$$
C_{*}\left(W^{\prime}, \partial_{0} W ; Z\left[\Gamma_{n+1}\right]\right) \rightarrow C_{*}\left(W^{\prime}, \partial_{0} W ; Z\left[\gamma_{n+1, n}\right]\right) \stackrel{\hat{f}}{\longrightarrow} C_{*}\left(W, \partial_{0} W ; Z\left[\gamma_{n+1, n}\right]\right) .
$$

Recall (4.3) that $\operatorname{Sc}\left(Z\left[\Gamma_{n+1}\right]\right) \downarrow D_{*}$ is defined when $D_{*}$ is a $Z\left[\Gamma_{n+1}\right]$ complex whose $Z\left[\Gamma_{n}\right]$ reduction is a small chain object. This set is the equivalence classes of $\left(E_{*}, q\right)$, where $E_{*}$ is a small chain object over $Z\left[\Gamma_{n+1}\right]$ and $q: E_{*} \rightarrow D_{*}$ is a chain map whose $Z\left[\Gamma_{n}\right]$ reduction is a simple equivalence.

Note that the identity homomorphism on $\Gamma_{n}(W)$ and the identity map on chains defines a homological equivalence of $M \cup W$ to itself. This has a chain invariant, namely $\left(C_{*}\left(W, \partial_{0} W ; Z\left[\Gamma_{n+1}\right]\right), q\right)$, where $q$ is the change-of-coefficient map to the stratified chains. We will say the chain invariant of $(\phi, f)$ is trivial if it is the same as the invariant of the identity. Note the sets in which the chain invariants take values have not been given group structures, and even if they have group structures "trivial" may not be the same as "=0". It seems reasonable to hope that these sets have affine structures (free effective action of an abelian group) so choosing a basepoint gives an abelian group structure with this point as 0 . In this case choose the invariant of the identity as the basepoint, and "trivial" becomes the same as " $=0 "$ ". The $K$-theory speculation of 5.3 is in part an effort to find an affine structure.

6.4 Theorem. Suppose $M \cup W$ is a $\Gamma$ decomposition of $N$.

(1) Every element of $\operatorname{Sc}\left(Z\left[\Gamma_{n+1}\right]\right) \downarrow C_{*}\left(W, \partial_{0} W ; Z\left[\gamma_{n+1, n}\right]\right)$ is realized as $\tau_{n+1}$ of a homological $\Gamma_{n}$ equivalence;

(2) $\tau_{n+1}$ of a homological $\Gamma_{n}$ equivalence is unchanged by composition with a homological $\Gamma_{n+1}$ equivalence, so in particular is unchanged by $\Gamma_{n+1}$ deformation, or $\Gamma$ grope deformation of height $n+2$. 
(3) Suppose $(\phi, f)$ and $(\lambda, g)$ are homological $\Gamma_{n}$ equivalences to decompositions $M^{\prime} \cup W^{\prime}$ and $\widehat{M} \cup \widehat{W}$, respectively. Then there is a homological $\Gamma_{n+1}$ equivalence $(\alpha, h)$ from $M^{\prime} \cup W^{\prime}$ to $\widehat{M} \cup \widehat{W}$ with $\alpha$ the canonical isomorphism on $\Gamma_{n+1}$ and $h$ a chain equivalence lifting the $\Gamma_{n}$ equivalence $g f^{-1}$, if and only if $\tau_{n+1}(\phi, f)=\tau_{n+1}(\lambda, g)$.

Proof. This follows easily from previous results and definitions. Statement (1) comes from 4.4. The first part of statement (2) is a consequence of the equivalence relation used in the definition of $\operatorname{Sc}\left(Z\left[\Gamma_{n+1}\right]\right) \downarrow C_{*}\left(W, \partial_{0} W ; Z\left[\gamma_{n+1, n}\right]\right)$, and the second part comes from the fact (3.4) that deformations induce chain equivalences. Finally (3) is again a restatement of the definition of the equivalence relation on chain invariants.

6.5 The obstruction theory. Suppose $M \cup W$ and $M^{\prime} \cup W^{\prime}$ are $\Gamma$-decompositions of $N$. Theorem 6.3 gives a plan for comparing them: first see if they are homologically $\Gamma$ equivalent. If not, they are different. If so, choose an equivalence $\left(\gamma_{0}, f_{0}\right)$ and consider the chain invariant $\tau_{1}\left(\gamma_{0}, f_{0}\right)$. If this is nontrivial (different from the invariant of the identity), then the decompositions are at least somewhat different. If the invariant is trivial, then there is a homological $\Gamma_{1}$ equivalence. Continue in the same way: at each stage we either see some difference or we proceed to the next stage. This plan suffers from the usual defects, which we now discuss. The hope is that the context here is sufficiently more explicit than the classical link setting that the defects can be better analysed.

6.5.1 Indeterminate. Triviality of an invariant $\tau_{n+1}$ means a $\Gamma_{n}$ equivalence lifts to a $\Gamma_{n+1}$ equivalence, but does not specify a particular lift. The invariant $\tau_{n+2}$ at the next stage depends on the choice of lift, so is not well defined as a function of the original data. The failure to be well defined can be related to chain self-equivalences of the chains of $\left(W, \partial_{0} W\right)$.

6.5.2 Inconclusive. Nontriviality of $\tau_{n+1}$ means a particular $\Gamma_{n}$ equivalence does not lift to a $\Gamma_{n+1}$ equivalence. It may be that some other $\Gamma_{n}$ equivalence does lift. Again this can be analysed in terms of chain self-equivalences of the chains of $\left(W, \partial_{0} W\right)$.

6.5.3 Limit problems. This scheme offers a way to compare $\Gamma_{n}$ covers of $\Gamma$ decompositions, for arbitrarily large $n$. We would like to be able to say that vanishing of all these invariants gives a homological equivalence of $\Gamma_{\infty}$ covers. The dependence on choices discussed above make even the approach unattractive, since "vanishing of all invariants" must be interpreted as "there is an infinite sequence of choices, each of which makes the next possible." But worse than that, the fact that there are choices means even if such a sequence is found there may be "lim." problems in fitting them together sufficiently well to pass to a limit.

6.5.4 Special cases. It may be possible to analyse chain self-equivalences, and therefore the problems above, in special cases where activity is largely confined to a single degree. Interesting examples are suggested in 5.3.2. 


\section{REFERENCES}

[CM] Tim Cochran and Paul Melvin, Finite type invariants of 3-manifolds, math.GT/9805026, Invent. Math. 140 (2000), 45-100. MR.1779798 (2002a:57015)

[COT] Tim Cochran, Kent Orr, and Peter Teichner, Knot concordance, Whitney towers, and $L^{2}$ signatures, math.GT/9908117, Ann. of Math. 157 (2003), 433-519. MR1973052 (2004i:57003)

[CT] James Conant and Peter Teichner, Grope cobordism of classical knots, Topology 43 (2004), 119-156. MR2030589 (2004k:57006)

[FQ] Michael Freedman and Frank Quinn, Topology of 4-manifolds, Princeton University Press, 1990. MR.1201584 (94b:57021)

[Q] Frank Quinn, Dual decompositions of 4-manifolds, Trans. Amer. Math. Soc. 354 (2002), 1373-1392. MR 1873010 (2002k:57080)

[R] Andrew Ranicki, Noncommutative localization in topology (to appear).

Department of Mathematics, Virginia Polytechnical Institute and State University, Blacksburg, Virgina 24061-0123

E-mail address: quinn@math.vt.edu 